\title{
Social integration predicts mitochondrial DNA copy number in rhesus macaques
}

\author{
Reena Debray*1, Noah Snyder-Mackler*2,3, Jordan Kohn ${ }^{4}$, Mark Wilson $^{4,5}$, Luis Barreiro ${ }^{6,7}$, \\ Jenny Tung $1,2,8$ \\ ${ }^{1}$ Department of Biology, Duke University, Durham, NC 27708, USA \\ ${ }^{2}$ Department of Evolutionary Anthropology, Duke University, Durham, NC 27708, USA \\ ${ }^{3}$ Duke Center for the Study of Aging and Human Development, Duke University, Durham, NC \\ 27708, USA \\ ${ }^{4}$ Yerkes National Primate Research Center, Emory University, Atlanta, GA 30322, USA \\ ${ }^{5}$ Department of Psychiatry \& Behavioral Sciences, Emory University, Atlanta, GA 30322, USA \\ ${ }^{6}$ Department of Genetics, Centre Hospitalier Universitaire Sainte-Justine Research Center, \\ Montréal, Quebec H3T1C5, Canada \\ ${ }^{7}$ Department of Pediatrics, Faculty of Medicine, Université de Montréal, Montréal, Quebec \\ H3T1J4, Canada \\ ${ }^{8}$ Duke Population Research Institute, Duke University, Durham, NC 27708, USA \\ *These authors contributed equally to this work.
}

\section{Author for correspondence:}

Jenny Tung

jenny.tung@duke.edu

Keywords: social status, social integration, primates, mitochondrial DNA, peripheral blood mononuclear cells, social stress 


\begin{abstract}
In many social mammals, social adversity predicts compromised health and reduced fitness. These effects are thought to be driven in part by chronic social stress, but their molecular underpinnings are not well understood. Recent work suggests that chronic stress can affect mitochondrial copy number, heteroplasmy rates, and function. Here, we tested the first two possibilities, for the first time in nonhuman primates. We manipulated dominance rank in captive female rhesus macaques $(n=45)$, where low rank induces chronic social stress, and measured mitochondrial DNA copy number and heteroplasmy in five peripheral blood mononuclear cell types from each study subject. We found no effect of dominance rank on either mtDNA copy number or heteroplasmy rates. However, grooming rates, a measure of affiliative social behavior predicted by high social status, was positively associated with mtDNA copy number in B cells, cytotoxic T cells, and monocytes. Our results suggest that social interactions can influence mtDNA regulation in immune cells. Further, they indicate the importance of considering both affiliative and competitive interactions in investigating this relationship.
\end{abstract}




\section{Introduction}

In social mammals, the rate and outcome of competitive and affiliative interactions often co-vary with fitness-related traits $(1,2)$. These relationships arise in part because social interactions influence access to other fitness-determining factors, such as food or mates, and in part because individual condition can shape social interactions. However, recent evidence also points to a third explanation: direct effects of social interactions on animal physiology, often in connection to socially induced stress (3). Socially subordinate or isolated individuals exhibit elevated glucocorticoid levels (4), up-regulated beta-adrenergic signaling (5), and altered gene regulation (6), which in turn may contribute to social gradients in disease and mortality rates $(7,8)$.

Recent evidence indicates that social adversity may also impact mitochondrial DNA (mtDNA) content, function, and regulation. Experimental studies in rodents indicate that chronic social stress reduces mitochondrial energetic capacity, alters mtDNA gene expression, and changes the complement of mitochondria-associated proteins and metabolites (9). Further, cortisol treatment induces glucocorticoid receptor binding to mtDNA (10), suggesting a link between hypothalamic-pituitary-adrenal axis regulation of stress and mitochondrial activity. Depression in women and physical stressors in mice also predict higher mtDNA copy number and heteroplasmy (mtDNA genetic variation within an individual) in blood and saliva $(11,12)$. Together, these findings suggest that mtDNA biology is sensitive to chronic stress, including social stress. However, this hypothesis has not been tested in nonhuman primates, despite extensive work on the consequences of social interactions for other measures of physiology, health, and fitness $(1,2,13)$.

Here, we address this gap by studying captive female rhesus macaques (Macaca mulatta), in which social status (i.e. dominance rank) was experimentally manipulated via controlled introduction of females into new social groups (14). Female rhesus macaques form stable, linear dominance hierarchies, and social status affects access to resources, social control, and exposure to psychosocial stress (15). In our experimental population, later introduction predicted lower rank, which in turn predicted higher rates of received harassment and lower rates of affiliative grooming behavior (14). To test the effects of social interactions on mtDNA biology, we measured mtDNA copy number and heteroplasmy in five purified peripheral blood mononuclear cell (PBMC) types. Motivated by previous observations in humans and mice $(11,12)$, we predicted that social subordination and relative social isolation - that is, increased exposure to social stressors-would lead to higher mtDNA copy number and heteroplasmy rates.

\section{Materials and Methods}

\section{a. Behavioral data collection}

We studied nine social groups, each composed of five unrelated adult female rhesus macaques (described in prior work (14): Table S1). Females were sequentially introduced such that later introduction predicted lower dominance rank. Rank was quantified based on agonistic behavioral interactions obtained during focal sampling (16) (121.5 hours of observation), using 
Elo ratings (17). To measure affiliative behavior, we used the proportion of focal observation time that a female spent grooming or being groomed.

\section{b. mtDNA copy number quantification}

DNA used in this study was obtained from previous work (14). In brief, PBMCs were purified from 12-20 mL of blood from each female, five PBMC cell types (B cells, cytotoxic $\mathrm{T}$ cells, helper T cells, monocytes, and natural killer cells) were sorted into separate populations, and RNA and DNA were extracted.

We measured mtDNA copy number in duplicate using quantitative PCR (qPCR), targeting a region of the mitochondrial genome and a single-copy region of the nuclear genome $(n=213$ samples for which sufficient DNA was available: see also Text S1). Using the cycle threshold (Ct)

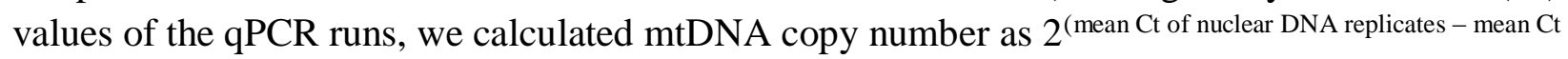
of mtDNA replicates), and log-transformed mtDNA copy number for downstream analysis. Our mtDNA copy number estimates were robust to use of alternative nuclear and mtDNA loci (Figure S1).

We modeled natural log-transformed mtDNA copy number as a function of social interactions (dominance rank or grooming behavior), controlling for age, cell type, and qPCR plate/batch (Text S2), using linear mixed effects models. We first investigated social interaction effects on mtDNA copy number across all cell types. Next, to test for heterogeneity in the effects of social interactions across cell types, we modeled each cell type separately and used a Bayesian meta-analytic approach to investigate shared effects across cell types (18).

\section{c. Heteroplasmic variants in $\mathrm{mtDNA}$}

We quantified mitochondrial heteroplasmy using mtDNA-mapped reads from previously generated, cell type-specific RNA-seq data for each study subject (14) (accession number GSE83307; see also Text S3) and the program mitoCaller (19). To account for sequencing coverage, we removed mtDNA positions with fewer than 200 reads and randomly subsampled sites with $>200$ reads down to exactly 200 reads. Following (12), we classified a site as heteroplasmic if the minor allele frequency was at least $4 \%$ within a sample.

We modeled heteroplasmy using a binomial mixed model approach (20), controlling for age and cell type. Our response variable was the number of heteroplasmic sites in the mtDNA genome relative to the number of analyzable sites for each sample.

\section{Results}

\section{a. Grooming behavior, but not dominance rank, predicts mtDNA copy number}

Dominance rank did not predict mtDNA copy number across all cell types together $(B=-$ 0.033, $p=0.931$, Table 1) or in any individual cell type (all $\mathrm{p}>0.1$, Table 1 ). However, mtDNA copy number was positively associated with the amount of time females spent grooming $(B=0.017$, $p=0.038$, Table 2). This association varied across cell types, with the strongest effect in B cells and a nonsignificant trend in monocytes and cytotoxic $\mathrm{T}$ cells (Figure 1). Consistent with these observations, formal meta-analysis strongly supported a shared positive effect of grooming on 
mtDNA copy number in B cells, monocytes, and cytotoxic $\mathrm{T}$ cells (Bayes factor=15.67). As expected (21), copy number varied systematically across cell types (Figure S2) and tended to decrease with age (Figure S3).

Table 1. Effects of dominance rank and age on mtDNA copy number.

\begin{tabular}{|c|c|c|c|c|}
\hline & Coefficient (B) & $\begin{array}{c}\text { Standard } \\
\text { error }\end{array}$ & Test statistic & $p$-value \\
\hline \multicolumn{5}{|l|}{$\underline{\text { All cell types }}$} \\
\hline Dominance rank & -0.033 & 0.038 & -0.087 & 0.931 \\
\hline Age & -0.017 & 0.011 & -1.660 & 0.099 \\
\hline \multicolumn{5}{|l|}{ Monocytes } \\
\hline Dominance rank & -0.010 & 0.073 & -0.138 & 0.891 \\
\hline Age & 0.016 & 0.019 & 0.822 & 0.420 \\
\hline \multicolumn{5}{|l|}{ Natural killer cells } \\
\hline Dominance rank & -0.048 & 0.098 & -0.491 & 0.626 \\
\hline Age & 0.014 & 0.027 & 0.512 & 0.611 \\
\hline \multicolumn{5}{|l|}{$\underline{B \text { cells }}$} \\
\hline Dominance rank & 0.036 & 0.074 & 0.486 & 0.630 \\
\hline Age & -0.032 & 0.020 & -1.633 & 0.110 \\
\hline \multicolumn{5}{|l|}{ Helper T cells } \\
\hline Dominance rank & -0.064 & 0.095 & -0.687 & 0.496 \\
\hline Age & -0.056 & 0.026 & -2.110 & 0.041 \\
\hline \multicolumn{5}{|l|}{ Cytotoxic T cells } \\
\hline Dominance rank & 0.059 & 0.078 & 0.768 & 0.447 \\
\hline Age & -0.052 & 0.024 & -2.158 & $\mathbf{0 . 0 3 7}$ \\
\hline
\end{tabular}

Table 2. Effects of grooming and age on mtDNA copy number.

\begin{tabular}{|c|c|c|c|c|}
\hline & Coefficient (B) & $\begin{array}{c}\text { Standard } \\
\text { error }\end{array}$ & Test statistic & $p$-value \\
\hline \multicolumn{5}{|l|}{ All cell types } \\
\hline Grooming & 0.017 & 0.008 & 2.086 & 0.038 \\
\hline Age & -0.018 & 0.010 & -1.774 & 0.078 \\
\hline \multicolumn{5}{|l|}{ Monocytes } \\
\hline Grooming & 0.028 & 0.015 & 1.821 & 0.078 \\
\hline Age & 0.013 & 0.017 & 0.749 & 0.462 \\
\hline \multicolumn{5}{|l|}{ Natural killer cells } \\
\hline Grooming & 0.011 & 0.019 & 0.561 & 0.578 \\
\hline Age & 0.008 & 0.026 & 0.321 & 0.750 \\
\hline \multicolumn{5}{|l|}{$\underline{B \text { cells }}$} \\
\hline Grooming & 0.035 & 0.015 & 2.308 & 0.026 \\
\hline Age & -0.033 & 0.018 & -1.838 & 0.073 \\
\hline
\end{tabular}




\begin{tabular}{|rcccc|} 
Grooming & -0.010 & 0.019 & -0.538 & 0.594 \\
Age & $\mathbf{- 0 . 0 5 9}$ & $\mathbf{0 . 0 2 6}$ & $\mathbf{- 2 . 3 0 3}$ & $\mathbf{0 . 0 2 7}$ \\
\hline Cytotoxic T cells & & & & \\
Grooming & 0.028 & 0.015 & 1.821 & 0.076 \\
Age & $\mathbf{- 0 . 0 5 2}$ & $\mathbf{0 . 0 2 2}$ & $\mathbf{- 2 . 3 6 1}$ & $\mathbf{0 . 0 2 3}$ \\
\hline
\end{tabular}

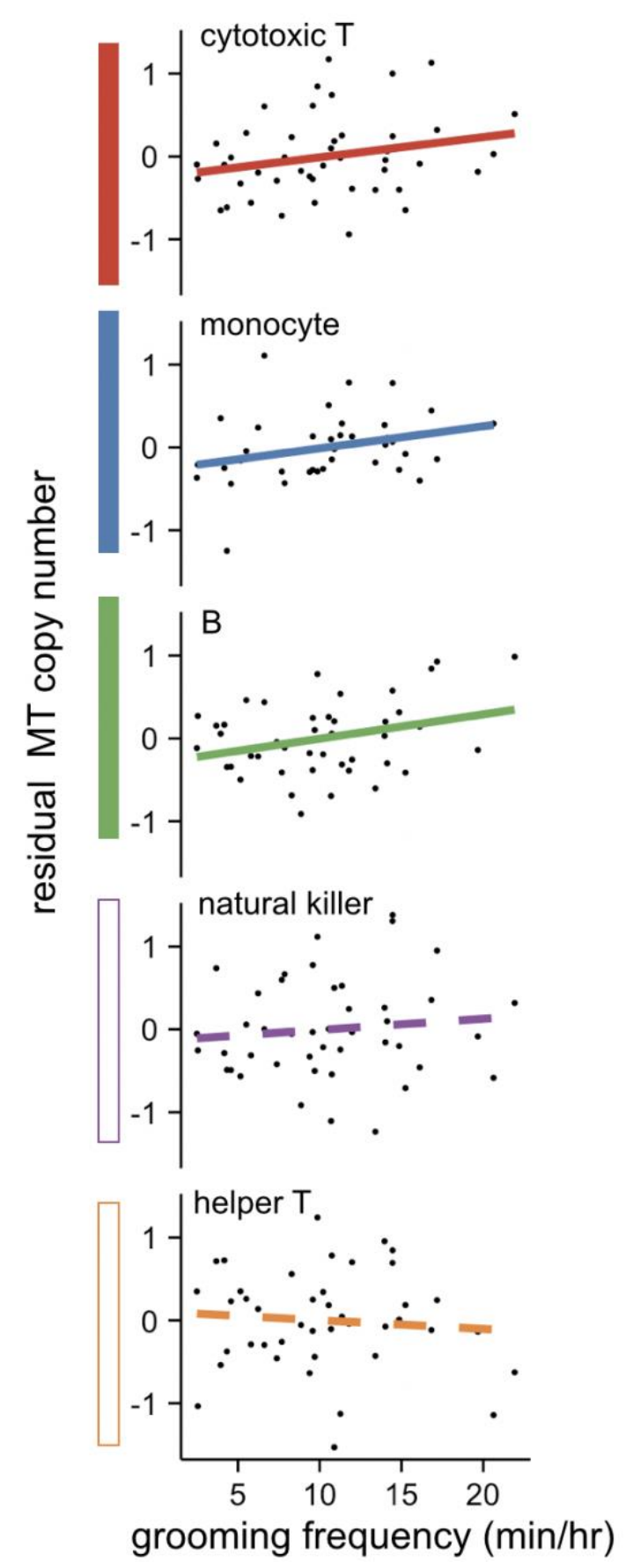

Figure 1. Cell type-specific effects of grooming on mtDNA copy number. Shaded boxes (left) show shared positive effects of grooming on mtDNA copy number based on meta-analysis (empty boxes $=$ no effect). For visualization purposes, the y-axis shows the residuals of a model controlling for age and qPCR batch. 


\section{b. Social interactions do not predict heteroplasmy}

Consistent with previous findings in humans (19), heteroplasmic sites occurred at low levels in each sample (mean number of heteroplasmic sites $=5.93 \pm 4.10$ s.d.). Most heteroplasmic mutations were private within samples, though samples from the same individual tended to share heteroplasmic sites (Figure S4). Neither dominance rank nor grooming rates were significant predictors of overall heteroplasmy rates (all p>0.1, Tables S4, S5, Figure 2).
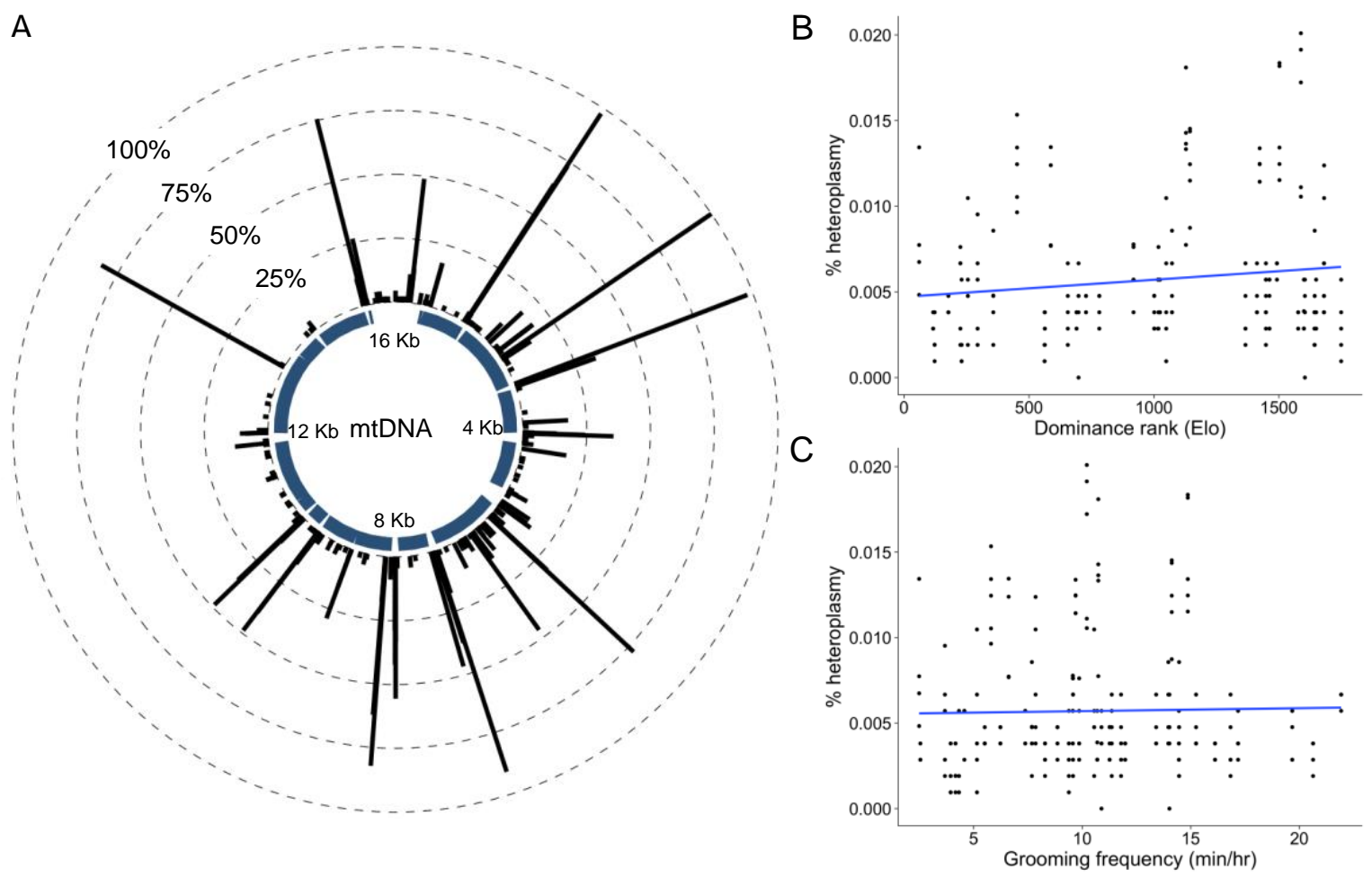

Figure 2. No effect of dominance rank or grooming on mtDNA heteroplasmy. (a) Locations and frequencies of heteroplasmic sites. The $\theta$-axis shows mtDNA coordinates based on (22). The y-axis shows the number of individuals (out of 45) that carried a heteroplasmy in at least one cell type. (b,c) Neither dominance rank (b) nor grooming behavior (c) predicts heteroplasmy rates, controlling for age and cell type.

\section{Discussion}

In contrast to our predictions, we found no effect of social status on mtDNA copy number. Further, high social affiliation was positively, not negatively, predictive of mtDNA copy number. Thus, our results support the idea that social interactions can influence mitochondrial biology, but they do so here in a surprising direction. One potential explanation for this difference is that previous studies focused on heterogeneous cell populations $(11,12)$, whereas here we analyzed purified populations. mtDNA copy number varies among cell types (21) (Figure S2). Thus, social factors that influence cellular composition could also affect apparent differences in copy number. Alternatively, low social status may affect mitochondrial biology differently than previously studied factors, such as early childhood adversity (23) or depression (11). We note, however, that our results are broadly consistent with findings that mtDNA copy number in the rat hippocampus 
and striatum was reduced by daily exposure to corticosterone and chronic exposure to mild physical stressors (24).

Together, our findings extend experimental studies of social factors on mitochondrial biology to primates, and add important data on females (10). We do not yet understand the importance of grooming effects on mtDNA copy number for cellular and organismal function. Increases in copy number may signify increased energetic potential or compensate for loss of mitochondrial function when some mtDNA copies are mutated. Additionally, while mtDNA copy number has been linked to many disorders, the directionality of this relationship depends on the disorder. For example, mtDNA copy number in blood is positively associated with rates of mitochondrial encephalomyopathies (26) but negatively associated with rates of metabolic syndrome (27).

Because this study used previously extracted DNA samples, we were not able to measure direct indicators of mitochondrial function. Future studies that measure both mtDNA copy number and mitochondrial metabolism in the same samples will clarify how mitochondrial quantity and quality combine to respond to social environmental cues. Such studies have the potential to shed new light on the relationship between mtDNA biology, human health, and its importance for Darwinian fitness in social mammals more broadly.

Acknowledgments. We thank J. Whitley, A. Tripp, N. Brutto, and J. Johnson for collecting behavioral data, N. Cai for advice on quantifying mtDNA copy number, and T. Reddy for access to the qPCR thermocycler.

Author contributions. JT, LBB, MEW, NSM, and RRD designed the study. RRD, NSM, and JK collected the data. RRD, NSM, and JT analyzed the data and wrote the paper, with contributions from all authors.

Data accessibility. Data are included as supplementary material, in SRA (accession number GSE83307), and at https://github.com/reenadebray/mtDNA_copy_number.

Funding. This work was funded by NIH grants 1R01-GM102562, P51-OD011132, and K99AG051764. RRD was supported by the Duke University Howard Hughes Research Fellowship and the Duke Undergraduate Research Support Office.

Competing interests. We declare no competing interests. 


\section{References}

1. Sapolsky RM. Social status and health in humans and other animals. Annu Rev Anthr. 2004;33:393-418.

2. Silk JB. Social Components of Fitness in Primate Groups. Science. 2007 Sep 7;317(5843):1347-51.

3. Creel S, Dantzer B, Goymann W, Rubenstein DR. The ecology of stress: effects of the social environment. Boonstra R, editor. Funct Ecol. 2013 Feb;27(1):66-80.

4. Creel S. Social dominance and stress hormones. Trends Ecol Evol. 2001 Sep;16(9):491-7.

5. Powell ND, Sloan EK, Bailey MT, Arevalo JMG, Miller GE, Chen E, et al. Social stress upregulates inflammatory gene expression in the leukocyte transcriptome via -adrenergic induction of myelopoiesis. Proc Natl Acad Sci. 2013 Oct 8;110(41):16574-9.

6. Tung J, Gilad Y. Social environmental effects on gene regulation. Cell Mol Life Sci. 2013 Nov;70(22):4323-39.

7. Silk JB, Beehner JC, Bergman TJ, Crockford C, Engh AL, Moscovice LR, et al. Strong and Consistent Social Bonds Enhance the Longevity of Female Baboons. Curr Biol. 2010 Aug;20(15):1359-61.

8. Shively CA, Register TC, Clarkson TB. Social stress, visceral obesity, and coronary artery atherosclerosis in female primates. Obesity. 2009;17(8):1513-20.

9. Picard M, McEwen BS. Psychological Stress and Mitochondria: A Systematic Review. Psychosom Med. 2018;80(2):141-53.

10. Hunter RG, Seligsohn M, Rubin TG, Griffiths BB, Ozdemir Y, Pfaff DW, et al. Stress and corticosteroids regulate rat hippocampal mitochondrial DNA gene expression via the glucocorticoid receptor. Proc Natl Acad Sci. 2016 Aug 9;113(32):9099-104.

11. Cai N, Chang S, Li Y, Li Q, Hu J, Liang J, et al. Molecular signatures of major depression. Curr Biol. 2015;25(9):1146-56.

12. Cai N, Li Y, Chang S, Liang J, Lin C, Zhang X, et al. Genetic control over mtDNA and its relationship to major depressive disorder. Curr Biol. 2015;25(24):3170-7.

13. Sapolsky RM. The Influence of Social Hierarchy on Primate Health. Science. 2005 Apr 29;308(5722):648-52.

14. Snyder-Mackler N, Sanz J, Kohn JN, Brinkworth JF, Morrow S, Shaver AO, et al. Social status alters immune regulation and response to infection in macaques. Science. 2016;354(6315):1041-5. 
15. Michopoulos V, Reding KM, Wilson ME, Toufexis D. Social subordination impairs hypothalamic-pituitary-adrenal function in female rhesus monkeys. Horm Behav. 2012;62(4):389-99.

16. Altmann J. Observational study of behavior: sampling methods. Behaviour. 1974;49(3):22766.

17. Albers PC, de Vries H. Elo-rating as a tool in the sequential estimation of dominance strengths. Academic Press; 2001.

18. Wen X, Stephens M. Bayesian methods for genetic association analysis with heterogeneous subgroups: from meta-analyses to gene-environment interactions. Ann Appl Stat. 2014;8(1):176.

19. Ding J, Sidore C, Butler TJ, Wing MK, Qian Y, Meirelles O, et al. Assessing mitochondrial DNA variation and copy number in lymphocytes of 2,000 Sardinians using tailored sequencing analysis tools. PLoS Genet. 2015;11(7):e1005306.

20. Lea AJ, Tung J, Zhou X. A flexible, efficient binomial mixed model for identifying differential DNA methylation in bisulfite sequencing data. PLoS Genet.

2015;11(11):e1005650.

21. Veltri KL, Espiritu M, Singh G. Distinct genomic copy number in mitochondria of different mammalian organs. J Cell Physiol. 1990;143(1):160-4.

22. Zimin AV, Cornish AS, Maudhoo MD, Gibbs RM, Zhang X, Pandey S, et al. A new rhesus macaque assembly and annotation for next-generation sequencing analyses. Biol Direct. 2014;9(1):20.

23. Tyrka AR, Parade SH, Price LH, Kao H-T, Porton B, Philip NS, et al. Alterations of mitochondrial DNA copy number and telomere length with early adversity and psychopathology. Biol Psychiatry. 2016;79(2):78-86.

24. Liu W, Zhou C. Corticosterone reduces brain mitochondrial function and expression of mitofusin, BDNF in depression-like rodents regardless of exercise preconditioning. Psychoneuroendocrinology. 2012 Jul;37(7):1057-70.

25. Barazzoni R, Short KR, Nair KS. Effects of aging on mitochondrial DNA copy number and cytochromec oxidase gene expression in rat skeletal muscle, liver, and heart. J Biol Chem. 2000;275(5):3343-7.

26. Liu C-S, Cheng W-L, Lee C-F, Ma Y-S, Lin C-Y, Huang C-C, et al. Alteration in the copy number of mitochondrial DNA in leukocytes of patients with mitochondrial encephalomyopathies. Acta Neurol Scand. 2006;113(5):334-41.

27. Huang C-H, Su S-L, Hsieh M-C, Cheng W-L, Chang C-C, Wu H-L, et al. Depleted leukocyte mitochondrial DNA copy number in metabolic syndrome. J Atheroscler Thromb. 2011;18(10):867-73. 
bioRxiv preprint doi: https://doi.org/10.1101/408849; this version posted September 6,2018 . The copyright holder for this preprint (which was not certified by peer review) is the author/funder, who has granted bioRxiv a license to display the preprint in perpetuity. It is made available under aCC-BY-NC-ND 4.0 International license.

28. Tamashiro KL, Sakai RR, Shively CA, Karatsoreos IN, Reagan LP. Chronic stress, metabolism, and metabolic syndrome. Stress. 2011 Nov;14(5):468-74. 